\title{
Implicaciones teóricas y prácticas de la adopción del Modelo de Servicio de Policía Comunitaria en Honduras
}

Theoretical and practical implications of the adoption of the Community Police Service Model in Honduras

\section{Implicações teóricas e práticas da adoção do Modelo de Serviço Policial Comunitário em Honduras}

\author{
Cristhian Eduardo Abate-Flores ${ }^{\mathrm{a}^{*}}$ | Óscar Urtecho-Osorto ${ }^{\mathrm{b}}$ Miguel Landa-Blanco ${ }^{\mathrm{c}}$ | Julio Ávila Flores ${ }^{\mathrm{d}}$ \\ | Luis Reyes Flores ${ }^{\mathrm{e}}$
}

a https://orcid.org/0000-000 I-7922-4207

${ }^{b}$ https://orcid.org/0000-000I-7404-6173

' https://orcid.org/0000-0002-7865-7593

${ }^{d}$ https://orcid.org/0000-0002-9228-6100

${ }^{\text {e }}$ https://orcid.org/0000-0002-5399-2766

Universidad Nacional de la Policía de Honduras, Honduras

- Fecha de recepción: 2020-0I-28

- Fecha concepto de evaluación: 2020-05-06

- Fecha de aprobación: 2020-05-13 https://doi.org//0.22335/rlct.v|2i2. I I 37

\begin{abstract}
Para citar este artículo / To reference this article / Para citar este artigo: AbateFlores, C. E., Urtecho-Osorto, O., Landa-Blanco, M., Ávila Flores, J., \& Reyes Flores, L. (2020). Implicaciones teóricas y prácticas de la adopción del Modelo de Servicio de Policía Comunitaria en Honduras. Revista Logos Ciencia \& Tecnología, 12(2), 85-96. https://doi.org//0.22335/rlct. v12i2. 1137
\end{abstract}

\section{RESUMEN}

El impulso de reformas institucionales de los cuerpos policiales en Latinoamérica ha traído consigo la implementación de nuevas prácticas que, en su mayoría, se orientan al fortalecimiento del vínculo policía-comunidad. En Honduras, desde el 2016 la Policía Nacional ha adoptado un Modelo Nacional de Servicio de Policía Comunitaria, en el marco de un proceso encaminado a cambiar la identidad policial, la cual históricamente ha estado más cerca de un ejercicio de la autoridad y el control frente a la comunidad $y$, en consecuencia, con la sociedad. El papel intersubjetivo que juega la comunidad en el ejercicio de la función policial se examina en el presente trabajo con el objetivo de explorar la naturaleza de la policía comunitaria. Esto y lo expuesto anteriormente hacen visible la necesidad de reconocer los retos y desafíos a superar para la implementación de un modelo de policía comunitaria en Honduras.

Palabras clave: policía, comunidad, cultura de paz, epistemología, sociología del cambio 


\begin{abstract}
The impulse of institutional reforms of the police forces in Latin America has brought with it the implementation of new practices that, for the most part, are aimed at strengthening the police-community bond. In Honduras, since 2016, the National Police has adopted a National Model of the Community Police Service, within the framework of a process aimed at changing the police identity, which historically has been closer to an exercise of authority and control over to the community. The intersubjective role that the community plays in the exercise of the police function is examined in this paper with the aim of exploring the nature of community policing. This and the aforementioned make visible the need to recognize and overcome the challenges in order to successfully implement the community policing model in Honduras.
\end{abstract}

Keywords: police, community, culture of peace, epistemology, sociology of change

\title{
RESUMO
}

O impulso das reformas institucionais das forças policiais na América Latina trouxe consigo a implementação de novas práticas que, em grande parte, visam fortalecer o vínculo polícia-comunidade. Em Honduras, desde 2016 a Polícia Nacional adotou um Modelo Nacional do Serviço Policial Comunitário, no âmbito de um processo destinado a mudar a identidade da polícia, que historicamente esteve mais próximo de um exercício de autoridade e controle frente a comunidade e, consequentemente, com a sociedade. $O$ papel intersubjetivo que a comunidade desempenha no exercício da função policial é examinado neste artigo com o objetivo de explorar a natureza do policiamento comunitário. Isso e o que já foi mencionado anteriormente tornam visível a necessidade de reconhecer os desafios a serem superados para a implementação de um modelo de policiamento comunitário em Honduras.

Palavras-chave: polícia, comunidade, cultura de paz, epistemologia, sociologia da mudança

Las reformas institucionales dentro de los cuerpos policiales han sido un fenómeno frecuente desde la década de los ochenta del siglo XX en América Latina. Estos cambios han mejorado las prácticas y la proyección de la policía hacia la ciudadanía (Ribeiro, Neiva \& Alves, 2016). En este sentido, las reformas están dirigidas a aspectos puntuales como la reducción de la violencia y la recolección de experiencias satisfactorias que mejoren el trabajo para el bien de la sociedad (Stone, 20I4). A fin de lograr esto es necesario el desarrollo de un modelo policial que permita ganarse la confianza de los ciudadanos, lo que implica separarse de la práctica basada en el castigo y el control, y pasar a la formación de una sensibilidad que facilite la vinculación con la comunidad por medio de la mutua escucha y la búsqueda conjunta de soluciones a la problemática que se enfrenta (García Gallegos, 2013).

La incorporación de elementos de vinculación comunitaria y la sensibilización de los entes del orden público, con respecto al rol de la ciudadanía como cogestora de su propia seguridad, se concretizan en el modelo de policía comunitaria, el cual tiene como fundamento, sobre todo, prácticas policiales estadounidenses. Estos nuevos procesos policiales tienen tres ejes: (a) la descentralización en la toma de decisiones, (b) la colaboración de la comunidad en los mapeos delictivos y (c) el empleo de estrategias basadas en la solución de problemas (Skogan, 2008). La implementación de un modelo así requiere un cambio cultural de gran envergadura $y$, por tanto, la renovación misma del sistema de educación policial, reconstruirlo a partir de las nuevas capacidades que se quieren formar.

Toda construcción educativa conlleva la condensación estructural de posturas ontológicas y epistemológicas aplicadas en el diseño y el desarrollo del programa en cuestión. Estas elaboraciones deberían ser particularmente notables en la educación policial y, por extensión, en el actuar operativo de los policías formados por tal sistema. En el caso de Honduras, desde el 2016 la Policía Nacional ha adoptado un Modelo Nacional de Servicio de Policía 
Comunitaria (Secretaría de Estado en el Despacho de Seguridad, 2016). Este evento ha reformulado las necesidades y las expectativas respecto a la formación inicial y continua de los miembros del cuerpo policial, lo cual está repercutiendo en la operatividad profesional. En este escenario es de suma importancia discutir las implicaciones del modelo comunitario que se lleva a la práctica en el campo policial en Honduras.

\section{Aspectos generales del Modelo Nacional de Servicio de Policía Comunitaria}

El modelo de policía comunitaria es un arquetipo gestado y articulado por equipos multidisciplinarios orientados al campo de la seguridad ciudadana con el propósito de salvaguardar el orden y la armonía social (Pandey, 20l4). Este modelo toma como base la relación policía-comunidad, asumiendo una interacción constante, inmediata y cooperativa (Stergioulis, 2018). Su fundamentación filosófica tiene implicaciones pragmáticas y fomenta estrategias organizacionales integradas por tres elementos: (a) vínculo comunitario, (b) transformación organizacional y (c) solución de problemas (Community Oriented Policing Services, 20I4).

Los tres factores expuestos plantean una colaboración dinámica y bidireccional entre la policía y la comunidad. Esta simbiosis social implica que en el seno de la policía debe llevarse a cabo una renovación institucional -implementada desde el sistema de educación y que impacte la cultura policial- que permita la generación de soluciones a los problemas que enfrenta la comunidad mediante la integración de los ciudadanos en la búsqueda de estas alternativas. Con el fin de comprender este vínculo fundado en un objetivo común es vital considerar la comunidad como una estructura social compleja y evolutiva, entre cuyos miembros es necesario que exista confianza en el accionar policial para garantizar la paz y la seguridad (Alvarado, 20I0).

Asumiendo esta complejidad cambiante, toda vinculación policía-comunidad debe integrar a los diversos actores interesados, entre los cuales se encuentran agencias gubernamentales no policiales como escuelas, instituciones de salud y fiscalías. Otros involucrados son los grupos e individuos que convergen en la comunidad (voluntarios, líderes, activistas, habitantes, visitantes y turistas, entre otros). Además, se debe realizar un acercamiento con organismos no gubernamentales y proveedores de servicios entre los que se incluyen grupos religiosos y organizaciones civiles.
Finalmente, el vínculo policía-comunidad también tiene que considerar a las empresas privadas y los medios de comunicación (Community Oriented Policing Services, 20I4).

En el caso particular de Honduras se han identificado prácticas prometedoras en el abordaje policial comunitario dirigidas a generar vínculos con la ciudadanía (Barak, Lowrey-Kinberg, Maguire \& Hernández, 2018). Tales prácticas incluyen la participación en eventos comunitarios como actividades deportivas, brigadas médicas, proyección de películas en áreas públicas y recreovías Estas últimas son actividades en las que la policía se involucra a fin de crear espacios de sana convivencia, paz y actividad física para los ciudadanos, fomentado así una cultura libre de violencia y hábitos de vida saludable a través del ejercicio (Organización Panamericana de la Salud, 2019).

La policía comunitaria hondureña también participa en actividades que se dan en espacios escolares mediante la implementación de programas como Educación y Entrenamiento sobre Resistencia a Pandillas (Great, por sus siglas en inglés). En estas intervenciones se brinda capacitación a alumnos de escuelas priorizadas en temas de valores, prevención de violencia y consumo de alcohol y drogas, entre otros. Un tercer tipo de actividad comunitaria se refiere a los patrullajes "Meet-N-Greet", así como visitas a hogares, negocios y asociaciones de transportistas. En esta forma de intervención se espera que el policía se presente ante la comunidad y comparta sus datos de contacto, escuche activamente la problemática que enfrentan los ciudadanos, planifique acciones consensuadas y ofrezca recomendaciones respecto a la denuncia de delitos (Barak et al., 20I8).

\section{La construcción social del} vínculo policía-comunidad

Dadas las implicaciones del modelo comunitario existen eventos con características subjetivas en cuyo análisis es necesario considerar los puntos de vista de los actores. Un ejemplo de esto es la convivencia, construida sobre la base de las percepciones individuales, los imaginarios colectivos e, incluso, las aspiraciones y los deseos de los miembros de una comunidad. Debido a esto, el análisis de este fenómeno amerita profundizar en el saber que los miembros del grupo tienen sobre los otros y en los aspectos que facilitan o dificultan su mutua comprensión, un 
factor que a su vez contribuye a integrar las sociedades humanas (Scheler, 20I5).

En este caso, el "objeto" del abordaje policial es en realidad un "sujeto" pensante, cambiante, reactivo y emotivo. De manera que debe considerarse que la "realidad" en que se vincula con sus pares es fruto de un constructivismo social, es una edificación dinámica entre actores (Berger \& Luckmann, 1999). Esta construcción social se genera a partir del pluralismo cultural que implica el mundo y la comunidad -en cuanto obra grupal e incluso en su ser natural-, percibidos de forma distinta por individuos y colectivos, pues cada uno los mira desde su particularidad (Habermas, 2003).

A fin de abordar y garantizar la convivencia pacíica de una comunidad, así como de estudiar los conflictos sociales que se suscitan en ella, es de vital relevancia considerar este constructivismo social. Así, por ejemplo, en una discusión entre vecinos cada parte construye, percibe $y$, por ende, actúa según su propio marco de referencia. La incongruencia expresa de estas realidades puede resultar en un conflicto social mayor que atente contra la buena convivencia en la comunidad. Los elementos que subyacen en conflictos de convivencia de este tipo, así como la participación activa en la solución, requieren un policía educado científica y humanísticamente para comprender los diferentes puntos de vista que están en juego y actuar con empatía ante los actores (Feyerabend, 2003), en la búsqueda de la conciliación social. En este proceso el policía se convierte, además, en un constructor colaborativo de la realidad social, en un actor que busca una solución al conflicto; por tanto, es una tercera perspectiva ante la problemática que enriquece el escenario social en su papel de garante de la paz y el orden.

En esta interacción constructiva de la realidad social el policía comunitario cumple un doble papel. Por una parte, es un miembro más de la comunidad o se ha acercado a ella de tal manera que la entiende a través de su propio imaginario, en cuya construcción la misma comunidad ha permeado, al menos, algunos de sus patrones culturales, por lo que está vinculado a los ciudadanos y al espacio comunitario por una relación de empatía. Por otra parte, se encuentra su papel de autoridad legalmente constituida. El poder que le confiere esta legalidad, dada su condición de policía comunitario, se ubica más allá del plano del control: está en su condición de árbitro y colaborador en la búsqueda de la seguridad de las personas, quienes han depositado en él su confianza. De esta manera pasa de detentar el poder que le confiere el Estado a ejercerlo en la práctica como una forma de dominación consensuada en beneficio de los ciudadanos (Foucault, 2018).

El poder de la policía comunitaria, en consideración a la construcción de la realidad social expuesta hasta aquí, radica en la aceptación de su autoridad como válida por parte de la comunidad, la cual, a su vez, ejerce un poder -en un evento de libertad de elección gratificante que coadyuva a la paz- al depositar su confianza en la autoridad policial y en someterse a ella con base en esa confianza (Deleuze, 1987). A partir de este punto de apoyo se configura una relación con un objetivo común: garantizar el orden y el bienestar de las personas. La coincidencia en esta meta hace que el accionar policial se considere justo $y$, por tanto, reduce las posibilidades de manifestaciones sociales de inconformidad que pueden poner en riesgo los cimientos mismos de la sociedad (Laski, 20I3).

En la relación descrita, el antisocial se percibe como una amenaza para la comunidad $y$, por ende, como un sujeto intrusivo contra el que es necesario aplicar correctivos. El establecimiento y la validación de estos papeles se desarrolla mediante la proximidad policía-comunidad, en un proceso que permite la elaboración de un sentido compartido de la realidad en el que uno y otro actor se consideran aliados ante una problemática que afecta el escenario en el que conviven como miembros de un mismo grupo (Husserl, 1997).

Si bien es cierto que el vínculo policía-comunidad tiene lugar en una realidad social construida en colaboración, la concreción práctica de este vínculo se realiza en el contacto con el sujeto, quien se convierte en el centro del accionar de la policía comunitaria. Esto impele al policía a conocer los problemas que le atañen en la comunidad, pero también a centrarse, con mirada preventiva, en los individuos más vulnerables producto de su posición en la estructura económica y social (Touraine, 2013). Esta vulnerabilidad está relacionada con la posibilidad de que sean víctimas del delito o participantes en él, de tal manera que el cuerpo policial, en su papel comunitario, se ve obligado a conocer las causas generadoras de inseguridad y violencia, de tal manera que su trabajo preventivo sea focalizado, inteligente y efectivo a fin de que se refleje en las condiciones de vida de la comunidad. 
En consonancia con la realidad descrita, el modelo de policía comunitaria plantea un abordaje sistémico multidimensional en el que se admite que ni la comunidad ni la policía gozan de independencia una de la otra. Esta interdependencia da lugar a la conformación de un sistema social complejo cuya naturaleza depende de las transformaciones que sufren sus miembros al vincularse y no simplemente de la suma de cada uno de ellos. Así, producto de la vinculación de la policía con la comunidad, concebida a partir de la proximidad, se genera una nueva forma de convivencia en la que ambos actores se transforman para dar lugar a una estructura comunitaria fortalecida (Durkheim, 20I5). A raíz de esto, la mirada policial debe centrarse en el sujeto como objeto inmediato de su accionar, pero el análisis de su problemática tiene que realizarlo a partir de la estructura social comunitaria, pues es un error metodológico que imposibilita la compresión ontológica de un sistema complejo reducir el análisis a sus partes aisladas o a la simple suma de ellas (Morin, 2009).

\section{La construcción simbólica de la nueva}

\section{identidad policial comunitaria}

En la medida en que la Policía Nacional asume esta complejidad sistémica se vuelve necesario realizar cambios a nivel de la identidad y la formación policial. De esta manera, la aplicación de un modelo de servicio de policía comunitaria admite que el propio concepto y la operatividad del abordaje comunitario implica la construcción de un nuevo elemento doctrinal dentro de la cultura policial (Policía Nacional de Honduras, 2016). En este punto es necesario agregar que la operatividad policial tradicional implica que el policía, en referencia a la comunidad, es un actor distante, hermético, reactivo y externo. Por contraste, el modelo de policía comunitaria conlleva de forma inherente un principio de cercanía (Policía Nacional de Honduras, 2016). Debido a esta proximidad se espera que el policía sea un actor proactivo en todo espacio relativo al bienestar comunitario, de forma que su presencia como colaborador sea permanente, en lugar de realizar solo apariciones reactivas y esporádicas.

La adopción de un modelo comunitario, si se considera lo expuesto hasta aquí, requiere que el mismo policía construya $\circ$ reestructure su noción identitaria, pasando de una identidad autoritaria a un policía que se identifica a sí mismo como un constructor de convivencia y desarrollo comunitario, en conjunto con los demás actores sociales involucrados. Esto, esencialmente, implica la adopción de una postura constructivista aplicada a una diversidad de problemas comunitarios. Esta renovación del ser policial, que tiene un impacto directo sobre el trabajo operativo de los individuos, empieza necesariamente desde la institucionalidad misma de la policía, en la manera de concebir la formación académica del recurso humano, con una renovación cultural que permita la asunción de liderazgos acordes al nuevo contexto social y la reconstrucción de la imagen y la autoimagen del policía (Eidelman, 20I2).

La reconfiguración de la identidad del policía comunitario se va conformando con respecto a la comunidad de pertenencia. Toda comunidad tiene códigos, valores y patrones culturales (Baudrillard, 1980), de modo que, en la medida en que el policía se acerca y se identifica con ella, los conoce, asume e interioriza de manera progresiva $y$, cuando es necesario, ayuda a redefinirlos. De estas capacidades depende, en gran medida, el impacto que tiene sobre los ciudadanos. Debido a esto, además, el policía comunitario no solo es operativamente activo, sino también intelectualmente. Se identifica y piensa los problemas desde la comunidad, pero también deber ser capaz de autopercibirse y ser considerado por los otros como un líder con influencia y derecho para actuar en nombre del resto de los ciudadanos.

Esta proximidad, en esencia, plantea una mutua cercanía entre actores. Consecuentemente, es necesario comprender las percepciones de la policía hacia la comunidad y viceversa. En el primer caso, si bien tiene un papel de liderazgo moral y operativo, el policía debe identificarse como parte de la comunidad, en particular al considerar la naturaleza civil de la institución a la que pertenece. De esta forma, no debe existir una distinción identitaria entre "policía" y "ciudadano", ya que la pertenencia a la carrera policial no implica la separación del atributo ciudadano. Se trata entonces de establecer una nueva forma de vinculación entre iguales que permita fortalecer el tejido social comunitario mediante la corresponsabilidad y el trabajo conjunto (Policía Nacional de Honduras, 2016). Por consiguiente, asumir un papel comunitario requiere de un cambio en el autoconcepto policial y, extensivamente, en su cultura.

Por otra parte, al considerar la percepción de la comunidad hacia la policía, se evidencia la relevancia de la confianza en la institución. La necesidad de desarrollar esta forma de vínculo ha detonado múltiples reformas policiales, 
particularmente en países en vías de desarrollo (Goldsmith, 2005). En este contexto, a fin de incrementar la confianza institucional es particularmente útil transparentar los procesos de rendición de cuentas, evidenciar benevolencia, compartir un marco ético con la comunidad y realizar evaluaciones permanentes del trabajo policial (Bergman \& Flom, 2012). A esto se suma un uso ponderado de la fuerza, así como un trato respetuoso. Todo esto configura una identidad en la relación policía-ciudadano que se materializa en eventos, acciones y objetos portadores de significado (Baudrillard, 20I0).

Si se considera lo analizado hasta aquí, uno de los retos para la ideal implementación del modelo comunitario es la portación de armas por parte de los policías. En este sentido, desde la neurociencia se plantean estudios que sostienen cómo la exposición a un arma captura la atención visuoespacial con la misma efectividad que un estímulo amenazante (por ejemplo, una serpiente), en comparación con estímulos neutrales. Esto ofrece evidencia de la existencia de un efecto de incremento de la percepción de riesgo ante una persona que porta un arma (Fox, Griggs \& Mouchlianitis, 2007). Es decir, las armas se asocian al peligro (Ohman \& Mineka, 200I) y, en vista de esto, su portador también puede ser visto como peligroso o poco confiable.

Lo anterior puede crear una ambivalencia en intervenciones policiales comunitarias. Si bien es cierto que el arma es una herramienta en muchos casos indispensable para salvaguardar el bienestar de la ciudadanía, así como del mismo policía, su presencia como estímulo amenazante es capaz de generar reacciones de distanciamiento ante la figura policial. Considerando esto, y que el arma de fuego se ha relacionado históricamente con el ejercicio de una autoridad de control desde el aparato policial (Jar Couselo, 1999), su empleo como parte del equipo diario del policía comunitario promueve una verticalidad contraproducente para la construcción de vínculos bidireccionales idealmente horizontales entre policía y comunidad.

A fin de entender la trascendencia que un aspecto como este puede tener en el quehacer del policía comunitario, es válido analizar cómo han tratado la problemática en diferentes países. En el Reino Unido, por ejemplo, la fuerza policial se encuentra mayoritariamente desarmada $y$, cuando es necesario, emplea herramientas menos letales que las armas de fuego (College of Policing, 2019). Esto es posible gracias al modelo policial por consenso que se implementa en este país, en el que el poder de la policía es delimitado por el consentimiento del público, en lugar de ser impuesto por el Estado. Esto produce que el sentido del deber del policía esté orientado a la ciudadanía y no necesariamente al Estado (Yesberg \& Bradford, 2018).

La relación entre la policía y la comunidad es compleja y esto impacta la identidad de ambos. El modelo comunitario es un ejercicio de proximidad con la ciudadanía, el cual busca que esta renueve su confianza en la policía. En este sentido, para la comunidad el arma es un símbolo de la antigua forma de ser policía que implica un ejercicio distante, agotado como portador de significado positivo y, por tanto, rechazado. Buena parte de la labor del policía comunitario consiste en resignificarse de forma positiva ante la ciudadanía, por lo que debe construir nuevos símbolos que describan su relación con los ciudadanos. Sin embargo, la misma comunidad exige que el policía sea capaz de garantizarle seguridad impidiendo la libre acción de los delincuentes y castigándolos (Fondevila, 2009), y para esto es necesario que esté armado. ¿Cómo conciliar entonces la aplicación del modelo comunitario y la portación del arma de fuego?

En respuesta a este conflicto que plantea la construcción de la identidad en la relación policía-comunidad, en el Reino Unido se han creado unidades especiales armadas reactivas $-y$ diferenciadas del resto de los policías que hacen labor netamente comunitaria- con el propósito de actuar en situaciones que ameritan el uso de armas letales. En el caso particular de Escocia, un estudio reporta que el $53 \%$ de los encuestados están a favor de que un número limitado de policías visiblemente armados se involucren en actividades de rutina (TNS, 20I4). De forma complementaria, el $37 \%$ de la muestra se opuso a esta decisión y el restante $10 \%$ se mostraba indeciso sobre ella.

Al analizar los factores que determinan la aceptación o el rechazo de la ciudadanía hacía la policía armada se ha encontrado que la confianza juega un papel importante. Cuando los ciudadanos confían en la policía tienen más apertura a darle mayor poder dotándola de armas para el ejercicio de su autoridad. En contraposición, en los casos en que previamente se carece de este vínculo, el incremento del número de policías armados puede afectar de forma negativa el desarrollo de la confianza y los afectos de la ciudadanía hacia la institución (Yesberg \& Bradford, 2018). 
En otras palabras, además de crear nuevos símbolos que la vinculen con los ciudadanos, la policía comunitaria debe trabajar para resignificar la portación de armas como una prerrogativa que la comunidad le ha dado a partir de la confianza que tiene en ella. Esta resignificación empieza con una policía que se aproxima a la población de forma voluntaria, despojada del poder que le confiere el arma, hasta generar una identidad social única policía-comunidad, de tal manera que la primera sea vista como un recurso necesario para garantizar la convivencia pacífica de la segunda, protegiendo la integridad de las personas $y$ creando las condiciones para el fortalecimiento de valores colectivos que cohesionen a la sociedad (Illera, 2005).

Aquí surge una pregunta: ¿es posible un acercamiento de esta naturaleza independientemente del contexto social? Claramente, las condiciones sociales, institucionales, normativas y económicas de Reino Unido son diferentes a las de Honduras. Por ejemplo, para Inglaterra y Gales, la tasa de homicidios en el 2017 fue de 12,I por cada millón de habitantes (Office for National Statistics, 2018). Mientras que en ese mismo año la tasa de homicidios para Honduras fue de 43,6 por cada 100000 habitantes (Observatorio Nacional de la Violencia, 2018). De manera que la importación de un abordaje policial desarmado, aun con fines comunitarios, puede resultar contraproducente en términos operativos, inclusive riesgoso para el mismo cuerpo policial.

A partir de esto se evidencia que en Honduras las características propias del contexto obligan a que el policía porte arma de fuego, aun cuando esto puede verticalizar la relación con la comunidad. Por tanto, es particularmente necesario mejorar la confianza de la ciudadanía hacía la institución policial. Esta confianza implica, en sí misma, un reconocimiento ontológico de la necesidad de que el policía porte arma como estímulo disuasivo contra la delincuencia, y una lectura epistemológica de esto a partir de la concepción de la colectividad comunitaria como una unidad o sistema que se defiende a sí mismo, por medio de la policía, contra las amenazas criminales que agreden su integridad (Parsons, 1982).

\section{La intersubjetividad en el enfoque comunitario}

El enfoque comunitario tiene de fondo una filosofía humanista que impele a tomar en cuenta variables tradicional- mente invisibilizadas en el quehacer policial. Un ejemplo de esto, a propósito del principio de proximidad, es el del policía comunitario que debe preocuparse por el bienestar integral de los individuos. Esta integralidad $-y$, por ende, complejidad - implica que el policía debe tener no solo competencias operativas, sino también habilidades interpersonales funcionales, entre ellas que sea capaz de utilizar marcos de referencia ajenos al propio. A fin de lograr esto debe desarrollar una especie de imaginario sociológico que le permita entender cómo la comunidad está poblada por individuos de diversas psicologías, capacidades y papeles, y que el éxito de su trabajo depende de su compresión e integración propia en este espacio social (Wright Mills, 2017).

El desarrollo de esta capacidad empática tiene un carácter fenomenológico, desde el cual el sujeto puede tener un acercamiento comprensivo a la experiencia de un tercero. Tal elaboración es en sí misma una expresión de intersubjetividad (May, 20I7). En el ámbito policial, esta intersubjetividad puede permitir una mejor integración entre los diferentes actores que conforman la estructura comunitaria, que es un espacio supraindividual en el que los individuos se desarrollan y adquieren los significados que les dan identidad (Giddens, 2007). En este sentido, el abordaje fenomenológico permite la comprensión de la comunidad y sus actores en su propio contexto desde las observaciones empíricas (Bartkowiak-Théron, 2007).

Esta forma de conocer la comunidad le permite al policía, además, compartir las experiencias de los ciudadanos que la habitan $y$, por tanto, elaborar con ellos un ideario intersubjetivo, una consciencia del otro en cuanto diferente individualmente, pero con intencionalidades y motivaciones comunes en el espacio colectivo (Husserl, 2004). Adicionalmente, existe una estrecha asociación entre intersubjetividad y constructos tales como la compasión y la preocupación altruista hacia otros (May, 2017). En consideración a las características sociales y psicológicas propias del quehacer policial, la empatía puede ayudar a los policías a manejar situaciones de crisis e impactar en las interacciones sociales con la ciudadanía (Vinayak \& Judge, 2018).

En este sentido, entre las competencias que deben fomentarse se incluye la consciencia emocional de sí mismo y de otros. Esta empatía, idealmente, conlleva a la tolerancia, la aceptación y el respeto por la diversidad. Además de las características apreciativas de la empatía, la intersubjetividad se refleja expresivamente, de tal manera que el poli- 
cía es capaz de tener una comunicación efectiva, cálida y respetuosa con la ciudadanía (College of Policing, 2016). Por ende, si se considera que esta capacidad intersubjetiva-fenomenológica es un componente esencial de la labor policial comunitaria, es necesario reestructurar los planes formativos a fin de que estén orientados al desarrollo de estas competencias.

La educación científica del policía es de vital importancia porque le provee las herramientas necesarias para distanciarse conscientemente de la subjetividad en el abordaje de los problemas comunitarios. Si bien la intersubjetividad que lo vincula con el resto de la comunidad le permite identificar de forma objetiva los problemas que padecen los ciudadanos (Popper, 2017), es al poner en práctica la formación que ha recibido en la academia que puede deslindarlos de su apreciación subjetiva y operar sobre ellos para beneficio comunitario. Esta capacidad de discernimiento es trascendental si se considera que la comunidad es también un objeto de conocimiento al que el policía se acerca con preconcepciones que debe reelaborar. Esto lo realiza con base en un mecanismo experiencial y fenomenológico con la finalidad de aproximarse objetivamente a la realidad de los hechos sociales con los que tiene que trabajar (Bourdieu, 2007).

\section{El concepto de comunidad y el} modelo de policía comunitaria

La definición de comunidad en el modelo de policía comunitaria implica aspectos epistemológicos y ontológicos muy variados, así como un abordaje desde diferentes campos y enfoques conceptuales. En primera instancia, la comunidad se puede conceptualizar como un espacio geográfico en el que las personas interactúan con otras y se desenvuelven como entes sociales en plenitud (Krause Jacob, 200I). Esto implica la existencia de subespacios públicos y privados en los que códigos de conducta, normas legales y las mismas tradiciones regulan el comportamiento de las personas (Duhau \& Giglia, 2016). El policía comunitario tiene una existencia dual en estos espacios y subespacios: por una parte, se encuentra sujeto a sus reglas y patrones culturales; por otra, la comunidad le ha delegado la misión de garantizar que no se transgredan las normas que hacen posible la convivencia y el progreso social.

La definición expuesta, sin embargo, no agota la demanda de la sociedad contemporánea, en la cual la idea de comu- nidad se transmuta y adquiere elementos que la vuelven compleja. Las conceptualizaciones más recientes suponen la definición de comunidad a partir de dos elementos diferenciadores: la estructura y la funcionalidad. Desde lo estructural, la comunidad es vista por su delimitación geográfica y su organización política, social y cultural. La concepción funcional se refiere a las necesidades e intereses en común que un colectivo posee y a los mecanismos que se utilizan para suplirlas y alcanzarlos (Causse, 2009). En esta doble percepción convergen elementos puntuales que concretan la esencia de la comunidad: pertenencia, interrelación y cultura en común (Krause Jacob, 20l I). A partir de esta concepción el policía puede entender la comunidad como el espacio social al que pertenece, con cuyos ciudadanos interactúa y comparte una cultura.

A lo anterior cabe sumar que la comunidad también es una plataforma de encuentro de individualidades en la que la persona construye su ser social. En este marco de individualidades y elementos en común emerge un nuevo escenario que redefine la comunidad como un espacio de choque. De esta complejidad que adquiere la colectividad se desprenden conflictos de carácter interno, relacionados con los intereses particulares que se anteponen a los generales. Por ende, las instituciones encargadas del bienestar social, como la policía (Morales, Castellanos, Paz \& Rodríguez, 20I3), deben entender los espacios comunitarios en su calidad de sistema de convergencia de intereses y necesidades.

A fin de desempeñarse con éxito en un contexto así, el policía comunitario debe ser capaz de identificar la diferencia entre los hechos y las ideas que influyen en su interpretación (Galbraith, 1984), de tal manera que su actuar se perciba como objetivo y, por tanto, válido. Esto lo posibilita y lo enviste de la autoridad que le permite jugar un papel de mediador en la comunidad. Esta nueva figura que se antepone a la imagen autoritaria y coercitiva contribuye también a delinear la labor del policía comunitario, pues a partir de aquí su enfoque de acercamiento a la comunidad debe ser, sobre todo, preventivo (Cooper, 1997).

\section{Diversidad e igualdad en el modelo policial comunitario}

Anteriormente se argumentó el papel de la intersubjetividad empática en la labor policial comunitaria. Entre las manifestaciones de este constructo resaltó la mención al 
respeto por la diversidad. En este contexto, la comunidad, en cuanto estructura social compuesta, puede interpretarse según los principios de complejidad planteados por Morin (2009), en el sentido de que un sistema no tiene atributos sumativos sino transformativos. Es en esta conjugación diversa en la que la policía coexiste $y$, por ende, coadyuva a transformar la sociedad de la cual es parte.

Tal condición presenta un reto en la labor policial (Stergioulis, 2018), considerando que la igualdad y la diversidad son aspectos fundamentales de esta funcionalidad. La igualdad se plantea desde la premisa según la cual todas las características propias de la naturaleza humana merecen respeto. De esta manera, la sola tolerancia ante la pluralidad es insuficiente para lograr una integración transformativa entre los actores involucrados. Asimismo, se requiere que la policía practique, salvaguarde y acepte la diversidad, comprendiendo que la multiplicidad de estilos de vida, puntos de vista y opiniones, los contrastes e incluso la disensión son atributos característicos necesarios en una comunidad (Sartori, 20I5), y que uno de los trabajos policiales es colaborar en el propósito de lograr su coexistencia pacífica. Más aún, la diversidad debe considerarse un pilar de los derechos humanos (Stergioulis, 20l8). Esto incrementa la confianza de la ciudadanía, lo cual conlleva, como ya se dicho aquí, a una mayor aceptación y apoyo a la labor policial.

Siguiendo la línea de acción descrita, la Dirección Nacional de Educación Policial (DNEP) de Honduras ha plasmado en su visión institucional el desarrollo de competencias con enfoque de respeto a los derechos humanos, en el marco doctrinal del modelo comunitario (Dirección Nacional de Educación Policial, 2018). Esto se visibiliza en el diseño curricular del Técnico Superior No Universitario en Ciencias Policiales con Orientación en Seguridad Comunitaria, ofertado en el Instituto Técnico Policial (ITP) General José Santos Guardiola de Honduras, espacio donde se forman los miembros de escala básica. Su malla curricular contiene ejes de aprendizaje relativos a la formación jurídica y derechos humanos (fundamentos y aplicaciones), ciencias policiales con enfoque comunitario (fundamentos, patrullaje, gestión, enfoque de género y resolución de conflicto), estudios humanísticos y áreas complementarias (Dirección Nacional de Educación Policial, 2018). Cabe notar que el entrenamiento policial se considera fundamental para incrementar la sensibilidad ante la diversidad (Stergioulis, 20 I8).
También es fundamental la formación de líderes policiales capaces de guiar el proceso de acercamiento a la ciudadanía desde el modelo comunitario. Estos líderes deben tener una vocación de servicio profunda que los impela a anteponer el bien de los ciudadanos y el de sus compañeros ante cualquier cosa, incluso el propio bienestar. Su habilidad de ganarse el respeto de la comunidad debe estar sustentada en tratar a las personas cordialmente, como colaboradoras en su labor generadora de paz social (Aguilar Amaya, 2018). Esto supone el desarrollo, desde la formación en la academia policial, de una visión ontológica y epistemológica que les permita comprender holística y dialécticamente la comunidad como un todo complejo y cambiante, estructurado según múltiples puntos de vista que confluyen en una categoría intersubjetiva llamada "sociedad".

\section{Investigación-acción en el modelo de policía comunitaria}

Por la misma naturaleza del servicio comunitario, el policía debe tener formación en la implementación de modelos de investigación-acción (Bartkowiak-Théron, 2007). Estos métodos, per se participativos, permiten aproximaciones constructivistas a la realidad, así que pueden servir como base para el descubrimiento y la formación de nuevo conocimiento aplicado a la resolución de problemas. Sin embargo, su aplicación no se limita a la operatividad sino que también plantea un cambio en el paradigma policial para pasar de una verticalidad descendente, en la que las decisiones se toman desde los grados superiores hasta llegar a los grados de menor rango, a un modelo más horizontal de gestión de los recursos humanos policiales.

Esto se debe a que en el modelo policial comunitario es el policía operativo el que interactúa de forma directa con la comunidad, por lo que al aplicar la investigación-acción desarrolla un conocimiento de primera mano de los problemas presentes en dicho espacio. Tal policía, al ser el encargado de transmitir este saber, sirve de bisagra entre la institución y la comunidad. Debido a esto, sus superiores y la institucionalidad misma se ven obligados a reconocer su relevancia en cuanto a conocimientos, experiencias y relaciones interpersonales. De esta forma, el flujo de información dentro de la institución policial se vuelve, como mínimo, bidireccional. La escala básica pasa de ser un receptor de instrucciones para convertirse también en emisor de insumos relevantes dirigido a la construcción de intervenciones locales. En otras palabras, el policía 
operativo adquiere el poder de construir una imagen de la comunidad, basada en sus indagaciones y experiencias, así como de poner este objeto abstracto a disposición de sus superiores, quienes pueden tomar decisiones a partir de esto (Han, 2016). Se convierte en un vínculo entre la institución y la comunidad, y en este papel puede contribuir a configurar el destino de ambas.

Hacia la proyección comunitaria total: avances y desafíos de la policía en Honduras

La implementación del ejercicio policial comunitario, que empezó a discutirse formalmente en el país a partir de 1994 (véase la figura I), ha producido resultados positivos que han permitido a las fuerzas policiales hondureñas aumentar su efectividad y, por tanto, su credibilidad entre la población (esto a partir de una mejor comprensión de las preocupaciones de la ciudadanía en temas de seguridad). No obstante, lograr el desarrollo total del Modelo Nacional de Servicio de Policía Comunitaria supone un incremento en la cantidad de personal, equipamiento, entrenamiento policial y formación continua (Ungar \& Salomón, 2012), lo que aún es una meta a alcanzar en los próximos años a medida que avance el proceso de fortalecimiento de la institución en materia educativa, operativa y logística. Esto es necesario a fin de mejorar los mecanismos para la toma de decisiones, la colaboración en el combate al delito y la resolución efectiva de conflictos en el ámbito comunitario.

Otro gran reto que tiene el policía comunitario es el establecimiento de un vínculo de confianza que sirva como aprobación y validación de su trabajo por parte de la población, sobre todo en las comunidades pobres. En Latinoamérica, estas zonas históricamente han recibido poca atención del Estado, razón por la cual grupos delincuenciales como las maras han usurpado la labor policial de brindarles seguridad (Rodgers \& Baird, 20I6). El trabajo de la policía comunitaria consistió aquí, en un inicio, en retomar la capacidad de diálogo con la población. Luego, mediante el involucramiento en acciones y actividades de beneficio comunitario, poco a poco la policía construye una nueva identidad a partir del conocimiento sobre la comunidad. La meta aquí es recuperar el lugar que ha sido usurpado por otros grupos y convertirse en la única referencia para la población en materia de seguridad y garantías de convivencia.

La realización de lo expuesto amerita un cambio institucional y cultural complejo - y a veces traumático- que debe resolverse con el fin de desarrollar totalmente el

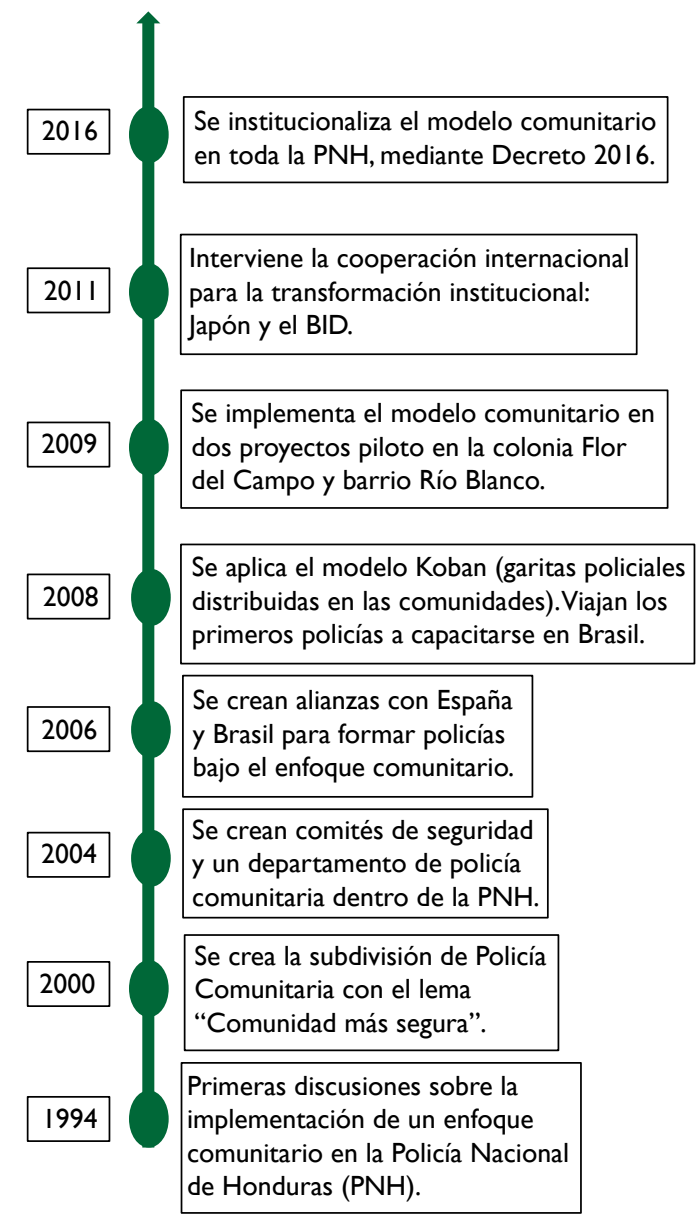

Figura I. Evolución histórica de la implementación del Modelo Nacional de Servicio de Policía Comunitaria en Honduras. Fuente: elaboración propia.

modelo de servicio comunitario en la institución. Por su naturaleza jerarquizada y porque posee una cultura profundamente enraizada, la renovación policial encuentra resistencias para asumir los nuevos roles en materia de liderazgo y operatividad que presupone el modelo comunitario, pues esto implica la pérdida del ejercicio tradicional de autoridad a cambio de una forma más democrática de ejercer influencia sobre la población (Hathazy, 20I6). La Policía Nacional de Honduras ha empezado esta renovación a partir del sistema educativo y la práctica del quehacer policial, en procura de adoptar como cultura los valores que implica la proximidad con la ciudadanía.

\section{Referencias}

Aguilar Amaya, M. (2018). Don de mando y liderazgo policial. Tegucigalpa: Universidad Nacional de Policía de Honduras.

Alvarado,A. (2010). Inseguridad pública, participación ciudadana y gobernanza. La ciudad de México en la última década. Estudios Sociológicos, 28(84), 94I-963. 
Barak, M., Lowrey-Kinberg, B., Maguire, E., \& Hernández, J. (2018). Identificando las prácticas de Policía Comunitaria potencialmente prometedoras en Honduras (Informe de consultoria, Unidos por la Justicia). Tegucigalpa.

Bartkowiak-Théron, I. (2007). The ethics of action research in community policing: the context of "no man's lands" and "no go áreas". En Annual AAPAE Conference. Melbourne: AAPAE. Recuperado de https://researchoutput.csu.edu. au/ws/portalfiles/portal/9625264/CSU266092.pdf

Baudrillard, J. (1980). El intercambio simbólico de la muerte. Barcelona: Monte Ávila Editores.

Baudrillard, J. (2010). Crítica de la economía política del signo. México: Siglo Veintiuno Editores.

Berger, P., \& Luckmann, T. (1999). La construcción social de la realidad. Buenos Aires:Amorrortu.

Bergman, M., \& Flom, H. (20I2). Determinantes de la confianza en la policía: una comparación entre Argentina y México. Perfiles Latinoamericanos, 40, 97-I 22.

Bourdieu, P. (2007). El sentido práctico. México: Siglo Veintiuno Editores.

Causse, M. (2009). El concepto de comunidad desde el punto de vista socio-histórico-cultural y linguístico. Ciencia en su PC, 3, | 2-2।.

College of Policing. (2016). Competency and values framework for policing: overview of framework. Reino Unido. Recuperado de www.college.police.uk/What-we-do/Development/ competency-and-values-framework/Documents/Competency-and-Values-Framework-for-Policing_4.I I.16.pdf

College of Policing. (2019). Code of Practice on Armed Policing and Police use of Less LethalWeapons. Recuperado de http:// library.college.police.uk/docs/appref/C6610319_Code_ of_Practice_Armed\%20policing_19.3.19.pdf

Community Oriented Policing Services. (2014). Community policing defined. Washington: U. S. Department of Justice. Recuperado de https://ric-zai-inc.com/Publications/copspl57-pub.pdf

Cooper, C. (1997). Patrol police officer conflict resolution processes. Journal of Criminal Justice, 25(2), 87- I0I. https:// doi.org/10.1016/S0047-2352(96)00053-0

Deleuze, G. (1987). Foucault. Barcelona: Paidós.

Dirección Nacional de Educación Policial. (2018). Plan de estudios delTécnico Superior en Ciencias Policiales con Orientación en Seguridad Comunitaria. La Paz.

Dirección Nacional de Educación Policial. (2018). Plan Estratégico de la Dirección Nacional de Educación Policial 20182020. Secretaría de Seguridad, Policía Nacional de Honduras, Tegucigalpa.
Duhau, E., \& Giglia,A. (2016). Metrópoli, espacio público y consumo. México: Fondo de Cultura Económica.

Durkheim, É. (2015). Las reglas del método sociológico. México: Fondo de Cultura Económica.

Eidelman, A. (2012). Construcción de la identidad policial y representación de la violencia política en la revista PFAMundo Policial durante los años 70. Contemporánea, 3(3), 53-75.

Feyerabend, P. (2003). Tratado contra el método. Espasa Calpe: Madrid.

Fondevila, G. (2009). Nuestra policía ideal. El Cotidiano, I53, 5I-56. Recuperado de http://www.redalyc.org/ pdf/325/325I5307.pdf

Foucault, M. (2018). Historia de la sexualidad: la voluntad de saber. México, D. F.: Siglo XXI.

Fox, E., Griggs, L., \& Mouchlianitis, E. (2007).The detection of fearrelevant stimuli: are guns noticed as quickly as snakes? Emotion, 7(4),69I-696. https://doi.org/I0.1037//528-3542.7.4.69 I

Galbraith, J. K. (1 984). La sociedad opulenta. Barcelona: Edaf.

García Gallegos, B. (20I3). Seguridad ciudadana y policía comunitaria en contexto de cambio político y social. Universitas, 19, 49-72. Recuperado de http://www.redalyc.org/ pdf/476 I/476 |47387002.pdf

Giddens, A. (2007). Las nuevas reglas del método sociológico: crítica positiva de las sociologías comprensivas. Buenos Aires: Amorrurtu.

Goldsmith, A. (2005). Police reform and the problem of trust. Theoretical Criminology, 9(4), 443-470. http://doi. org//0.1 I77//362480605057727

Habermas, J. (2003). La ética del discurso y la cuestión de la verdad. Barcelona: Paidós.

Han, B. C. (2016). La sociedad de la transparencia. Barcelona: Herder Editorial.

Hathazy, P. (2016). Democratización y campo policial. Nuevos consensos, cambios estructurales y mutaciones organizacionales en las policías de Chile (1990-2005). Civitas, I6(4), 595-617.

Husserl, E. (1997). Ideas relativas a una fenomelogía pura y a una filosofia fenomenológica. México: Fondo de Cultura Económica.

Husserl, E. (2004). Meditaciones cartesianas. México: Fondo de Cultura Económica.

Illera, M. (2005). Convivencia y cultura ciudadana: dos pilares fundamentales del derecho policivo. Revista de Derecho, 23, 240-259. 
Jar Couselo, G. (1999). El papel de la policía en una sociedad democrática. Reis, 85(99), 199-220.

Krause Jacob,M.(200I). Hacia una redefinición del concepto de comunidad: cuatro ejes para un análisis crítico y una propuesta. Revista de Psicología de la Universidad de Chile, 10(2), 49-60. https://doi.org/I0.5354/07/9-058I.2012.18572

Krause Jacob, M. (20I I). Representaciones sociales y psicología comunitaria. Psykhe, 8, 4I-47.

Laski, H. (20I3). Los peligros de la obediencia. San José: Uruk Editores.

May, J. (20I7). Empathy and intersubjectivity. En H. Maibom (Ed.), The Routledge handbook of philosophy of empathy (pp. 169-179). Nueva York: Routledge.

Morales, O., Castellanos, F., Paz, C., \& Rodríguez, X. (2013). La mediación en la resolución de conflictos: su interpretación desde la comunidad educativa. Interpretación y Perspectiva, 3(I), 70-93.

Morin, E. (2009). El método l: la naturaleza de la Naturaleza. Madrid: Ediciones Cátedra.

Observatorio Nacional de la Violencia. (2018). Boletín Nacional Enero a Diciembre 2017. UNAH. Tegucigalpa: IUDPAS. Recuperado de https://iudpas.unah.edu.hn/ dmsdocument/5880-boletin-nacional-enero-a-diciembre2017-ed-no-48

Office for National Statistics. (2018). Homicide in England and Wales: year ending march 2017. Recuperado de www. ons.gov.uk/peoplepopulationandcommunity/crimeandjustice/articles/homicideinenglandandwales/yearendingmarch2017/pdf

Ohman,A., \& Mineka, S. (200I). Fears, phobias, and preparedness: toward an evolved module of fear and fear learning. Psychological Review, 108(3), 483-522. https://doi.org//0.1037//0033295X.108.3.483

Organización Panamericana de la Salud. (2019). OPS Honduras. Recuperado de https:/www.paho.org/hon/ index.php? option $=$ com_content\&view $=$ article\&id $=1004:$ recreovias-paz-salud-honduras-estilos-vidasaludable\&ltemid $=225$

Pandey, V. (20/4). Community policing for conflict resolution and community resilience. International Journal of Social Work and Human Service Practice, 2(6), 228-233.

Parsons, T. (1982). El sistema social. Madrid:Alianza Editorial.

Policía Nacional de Honduras. (20I6). Guía de implementación del Modelo Nacional de Servicio de Policía Comunitaria. Tegucigalpa.
Policía Nacional de Honduras. (2016). Modelo Nacional de Servicio de Policía Comunitaria. Tegucigalpa.

Popper, K. (20I7). La lógica de la investigación científica. Barcelona:Tecnos.

Ribeiro, L., Neiva,V., \& Alves,A. (2016). Los significados de "policía comunitaria” para la Policía Militar Brasileña. Estudios Sociológicos, 34(I02), 603-627.

Rodgers, D., \& Baird,A. (2016). Entender las pandillas en América Latina: una revisión de la literatura. Estudios Socio-Juridicos, I8(I), I3-53.

Sartori, G. (2015). La democracia en 30 lecciones. México, D. F: Debolsillo.

Scheler, M. (2015). Sociología del saber ( $2^{\mathrm{a}}$ ed.). Buenos Aires: Leviatán.

Secretaría de Estado en el Despacho de Seguridad. (2016). Acuerdo Número 0267-2016. Oficio, Comayagüela.

Skogan,W. (2008). An overview of community policing: origins, concepts and implementation. Nueva York: John Wiley \& Sons.

Stergioulis, E. (2018). Police and diversity. The Police Journal: Theory, Practice and Principles, 9/(3), 264-274. https://doi. org/10.1 I77/0032258X17721136

Stone, C. (20I4). Dilemas de la reforma policial en contextos de alta criminalidad. En I. d. Públicos, Seguridad ciudadana en América Latina (pp. 9-17). Universidad de Chile.

TNS. (20I4). Police firearms survey. Recuperado de www.spa. police.uk/assets/I28635/tnssurveyreport

Touraine,A. (20I3). Después de la crisis. México, D. F.: Fondo de Cultura Económica.

Ungar, M., \& Salomón, L. (2012). Community policing in Honduras: local impacts of a national programme. Policing \& Society, 22(I), 28-42. https://doi.org//0.1080// 0439463.20 11.605136

Vinayak, S., \& Judge, J. (20/8). Impact of altruism and forgiveness on empathy among police personnel. Impact: International Journal of Research in Humanities, Arts and Literature, 6(2), 167-176.

Wright Mills, C. (2017). La imaginación sociológica. México, D. F.: Fondo de Cultura Económica.

Yesberg, J., \& Bradford, B. (2018).Affect and trust as predictors of public support for armed police: evidence from London. Policing and Society, I-19. https://doi.org//0.1080/104 39463.2018 .1488847 